\title{
A 38-Year-Old Pregnant Woman with Hemoptysis and Acute Renal Failure
}

\author{
Mateo Porres-Aguilar ${ }^{a}$ Juan B. Figueroa-Casas ${ }^{b}$ Mateo Porres-Muñoz ${ }^{c}$ \\ C. Gregory Elliott ${ }^{\mathrm{d}}$
}

${ }^{a}$ Department of Internal Medicine, Division of Respiratory Medicine, and ${ }^{b}$ Division of Respiratory and Critical Care Medicine, Texas Tech University Health Sciences Center/Paul L. Foster School of Medicine, El Paso, Tex., USA; 'Department of Internal Medicine, Beneficencia Española, Tampico, Mexico; ${ }^{\mathrm{d}}$ Department of Medicine, Division of Pulmonary and Critical Care Medicine, Intermountain Medical Center, Murray, Utah, and University of Utah Health Sciences Center, Salt Lake City, Utah, USA

\section{Case Report}

A 38-year-old Hispanic woman presented with a 4 -week history of hemoptysis of $20 \mathrm{ml}$ per day, fatigue, and bilateral arthralgias of her wrists and hands without joint swelling. She denied fever, chills, dyspnea or weight loss. Her past medical history was only remarkable for hypothyroidism. She was a nonsmoker and denied allergies, use of illicit drugs (cocaine, marihuana or heroin), recent travel or sick contacts. She had had 3 uncomplicated pregnancies and her last menstrual period had been 8 weeks prior to this presentation.

\section{Physical Examination}

She was pale, vital signs were normal and $\mathrm{O}_{2}$ saturation was $94 \%$ by pulse oximetry while breathing room air. Pulmonary examination revealed basilar crackles bilaterally. Cardiac examination revealed a 2/6 multifocal soft midsystolic murmur. The rest of the examination was unremarkable.

\section{Laboratory Findings}

Hemoglobin was $6.6 \mathrm{~g} / \mathrm{dl}$ with microcytosis and hypochromia. Blood urea nitrogen was $121 \mathrm{mg} / \mathrm{dl}$ and serum creatinine $10 \mathrm{mg} / \mathrm{dl}$. Both had been normal 13 days prior to her admission. Urinalysis demonstrated hematuria and dysmorphic red blood cell casts. A quantitative serum pregnancy test was positive. Bronchoalveolar lavage retrieved blood-tinged fluid, numerous red blood cells and hemosiderin-laden macrophages but few neutrophils. Microbiology studies for bacteria, mycobacteria and fungi in the bronchoalveolar lavage fluid were negative. Perinuclear antineutrophil cytoplasmic antibodies (P-ANCA) with specificity for myeloperoxidase (MPO) were positive $(5.5 \mathrm{U} / \mathrm{ml}$, normal $<0.4)$. Serologies for antiglomerular basement membrane, antinuclear antibodies, C-ANCA, antiproteinase 3 (PR3-ANCA), antinuclear antibodies, antiphospholipid panel, and HIV were negative.

\section{Radiographic Studies}

A chest radiograph was normal. High-resolution chest tomography showed bilateral ground-glass opacities distributed diffusely throughout both lungs (fig. 1).

\section{Biopsy Studies}

A renal biopsy showed organizing crescents, tubular atrophy and interstitial fibrosis (fig. 2). Immunofluorescence showed trace deposits of IgM along the capillary walls.

What is your diagnosis?

\section{KARGER}

Fax +4161306 1234

E-Mail karger@karger.ch

www.karger.com (c) $2011 \mathrm{~S}$. Karger AG, Basel

0025-7931/11/0821-0060\$38.00/0

Accessible online at:

www.karger.com/res
Mateo Porres-Aguilar, MD

Department of Internal Medicine, Division of Respiratory Medicine Texas Tech University Health Sciences Center/Paul L. Foster School of Medicine 4800 Alberta Ave., El Paso, TX 79905 (USA)

Tel. +1 915545 6618, E-Mail porres@email.com 
Fig. 1. High-resolution chest CT image on day 2 showing bilateral diffuse groundglass opacities.

Fig. 2. Renal biopsy showing organizing crescents, tubular atrophy and interstitial fibrosis.
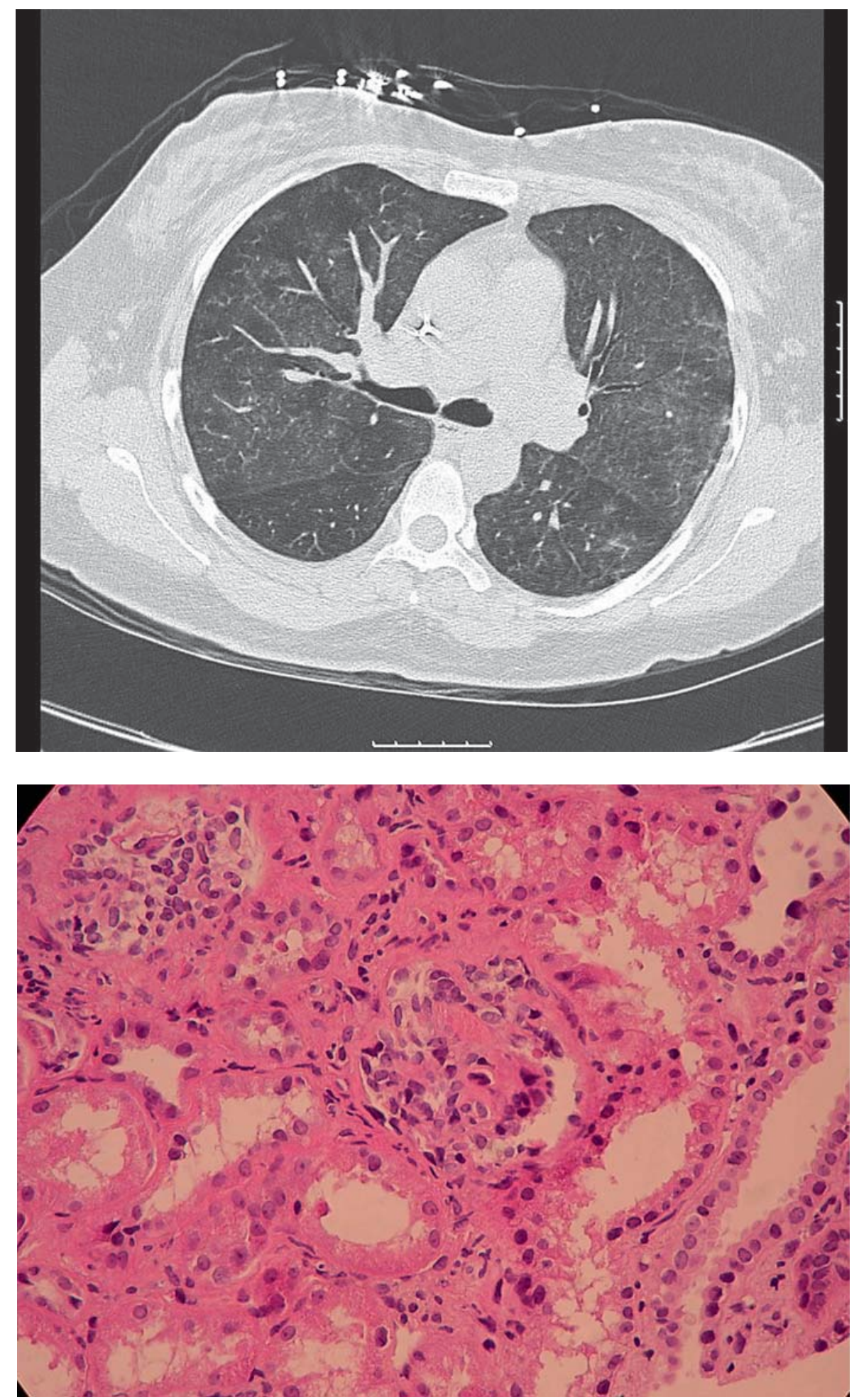


\section{Diagnosis: Microscopic Polyangiitis with Onset during the First Trimester of Pregnancy}

\section{Clinical Course}

Our patient was initially treated with intravenous methylprednisolone and plasma exchange therapy since her ANCA-associated vasculitis (AAV) was categorized as severe, generalized, life-threatening disease with at least 2 organs involved. The patient underwent successful dilatation and curettage after discussing the risks and benefits of continuing her pregnancy. Intravenous cyclophosphamide pulses were added after her surgical procedure. The patient is currently in remission, with complete recovery of her kidney function 9 months after discharge.

\section{Discussion}

Vasculitides associated with ANCAs are referred to as AAV. Microscopic polyangiitis (MPA) is an AAV characterized by pauci-immune necrotizing glomerulonephritis in more than $90 \%$ of cases and pulmonary capillaritis in up to $30 \%$ [1].

To our knowledge, only 1 case describing MPA with onset during pregnancy has been reported [2]. Milne et al. [2] reported a 25-year-old primigravida who presented at 24 weeks of gestation with 1 week of dyspnea and 2 days of hemoptysis. The patient was successfully treated with cyclophosphamide, systemic corticosteroids and plasmapheresis without the need for renal replacement therapy, and delivered a healthy girl at 35 weeks' gestation.

Two cases of pregnant women with previously diagnosed MPA have been reported. Schlieben et al. [3] described a woman with a history of MPA and severe preeclampsia, who became dyspneic and serologically active during the 33rd week of pregnancy. Forty-eight hours after delivery, the newborn developed diffuse alveolar hemorrhage and acute renal failure. The newborn's cord blood showed increased levels of MPO-ANCA identical to that in the mother's serum. Silva et al. [4] reported a case of transplacental transfer of high levels of MPO antibodies which did not result in clinical disease in the newborn of a mother with a 5-year history of MPA, who was in clinical remission prior to and during her pregnancy.

AAV presenting during pregnancy was first described in 1984 by Talbot et al. [5], who described a 26-week-pregnant woman who delivered a healthy 1,000-gram boy; a full recovery was made by the mother with corticosteroid and cyclophosphamide therapy. There have been 6 cases of Wegener's granulomatosis newly diagnosed during pregnancy [6]. Maternal and fetal death occurred in 1 case after cerebral hemorrhage. A therapeutic abortion was performed in 2 cases. In the 3 remaining cases, therapy with prednisolone and cyclophosphamide during the 2nd and 3rd trimester resulted in maternal and fetal survival, including the 1st case described by Talbot et al. [5].

We found a total of 14 reports in the current literature of women with a previous diagnosis of Wegener's granulomatosis who conceived during remission $[7,8]$. Relapse occurred during 3 pregnancies. Treatment of 3 patients included systemic corticosteroids alone, azathioprine combined with systemic corticosteroids and plasma exchange, and systemic corticosteroids and cyclophosphamide. All 3 mothers improved and delivered premature but otherwise healthy infants. Of the remaining 11 pregnancies with sustained remission, 1 pregnancy was terminated due to fetal encephalocele, the remaining women delivered healthy infants, with 3 of them being premature. Several reports exist of flares occurring immediately after either spontaneous or voluntary abortion, giving rise to the possibility that parturition brings immunologic changes that could predispose to disease flare [8].

It has been reported that the use of cyclophosphamide to treat severe life-threatening AAV during the 1st trimester of pregnancy has resulted in poor fetal outcomes, including spontaneous abortion or elective termination of pregnancy. Cyclophosphamide is an effective treatment for AAV, but is teratogenic, with a risk of congenital malformations during the organogenesis period $(<12$ weeks), causing spontaneous miscarriages and birth defects in up to $22 \%$ of fetuses $[7,8]$. Case reports regarding the use of this agent during pregnancy for neoplastic disorders suggest an increased risk of spontaneous abortions and various birth deformities when the mother is exposed to cyclophosphamide, particularly during the 1st trimester; however, neither accurate nor enough evidence exists of its use during severe flares of AAV during pregnancy, leaving the clinicians to discuss the risk/benefit ratio for treatment of relapsing AAV with cyclophosphamide - a difficult decision and a matter of debate - and to individualize the decision according to each patient's clinical characteristics [9].

Intravenous immunoglobulin (IVIG) represents an adjunctive mode of therapy that has been evaluated in relapses of $\mathrm{AAV}$, showing frequent induction of remission when standard agents are contraindicated, notably in the 
presence of an unacceptably high risk of infection or during pregnancy. Masterson et al. [10] described the successful induction of remission with IVIG and systemic steroids in a woman diagnosed with de novo Wegener's granulomatosis during the first trimester of pregnancy, offering the hope of the potential role of IVIG during pregnancy in the management of severe life-threatening AAV. Despite the case described above, evidence is lacking regarding the wide use of IVIG during pregnancy, plasma exchange therapy, as well as with targeted biological agents, making these alternative modes of therapy attractive for the design of future studies.

There is an important concern regarding the use and efficacy of IVIG in diverse forms of autoimmune glomerulonephritis, in particular the nephrotoxic effects of IVIG. Orbach et al. [11] performed a structured literature review from 1985 to 2003 and found 32 reports involving 78 patients with IVIG-induced nephrotoxicity whose data were compared with those of the 88 patients reported to the Food and Drug Administration. Most of the patients who developed renal toxicity had received sucrose-containing IVIG products as stabilizers. The mechanism of renal toxicity following IVIG use has not been clearly established; however, the histological changes from kidney biopsies, like vacuolization of proximal tubules, swelling and narrowing of the tubular lamina, are identical to those described in animal models following infusion of hypertonic sucrose, known as osmotic nephrosis [11]. The authors concluded that IVIG can be used safely in patients with various types of autoimmune glomerulonephritis; however, it is recommended that clinicians monitor renal function and be aware of risk factors that could predispose to renal toxicity, such as a rapidly progressive course, preexisting renal disease, volume depletion, dysfunction of other organs and age over 65 years.

Rituximab is a chimeric anti-CD 20 monoclonal antibody approved for the treatment of rheumatoid arthritis and non-Hodgkin's lymphoma. Recently, 2 multicenter, randomized, controlled trials were published comparing cyclophosphamide-based induction therapies versus rituximab in AAV, concluding that sustained remission rates were high in both groups, and in 1 of them the rituximab-based strategy was not inferior to daily cyclophosphamide therapy for induction of remission and may even be superior in relapsing disease [12,13].

Experience with rituximab during pregnancy is very limited. Neonatal B cell depletion has been reported following exposure at any stage of pregnancy. More studies are needed to assess potential neonatal complications that could be caused by transient $B$ cell depletion. Because of the lack of data, rituximab therapy should be stopped before conception $[14,15]$.

\section{Conclusion}

Microscopic polyangiitis is an AAV that universally involves the kidneys and involves the lungs in up to one third of cases. Systemic corticosteroids and cyclophosphamide represent the cornerstone of therapy in severe AAV; however, the addition of cyclophosphamide during pregnancy remains controversial and depends on the trimester; it should only be attempted after evaluating the risks and benefits for the mother and the fetus. Plasma exchange is indicated in the setting of severe and lifethreatening $\mathrm{AAV}$, demonstrating improvement in renal function; however, evidence-based data have been lacking during pregnancy. IVIG may be a therapeutic alternative in severe, refractory or life-threatening AAV when standard immunosuppressive agents entail an increased risk, notably during pregnancy; however, clinicians should be alert to the potential renal toxicity and patients at risk of developing osmotic nephrosis need to be identified. Biological agents like rituximab represent potential novel therapies for these particular clinical scenarios; however, further studies are needed to evaluate their safety for the neonate and mother before enrolling pregnant women in clinical trials.

\section{Key Words}

Microscopic polyangiitis - Pregnancy - Vasculitis - ANCA vasculitis $\cdot$ Pulmonary renal syndromes

References

$\checkmark 1$ Gomez-Puerta JA, Hernandez-Rodriguez J, Lopez-Soto A, Bosch X: Antineutrophilic cytoplasmic antibody-associated vasculitides and respiratory disease. Chest 2009;136: 1101-1111.

2 Milne KL, Stanley KP, Temple RC, Barker $\mathrm{TH}$, Ross CN: Microscopic polyangiitis: first report of a case with onset during pregnancy. Nephrol Dial Transplant 2004; 19:234237.

- 3 Schlieben DJ, Korbet SM, Kimura RE, Schwartz MM, Lewis EJ: Pulmonary-renal syndrome in a newborn with placental transmission of ANCAs. Am J Kidney Dis 2005; 45:758-761. 
4 Silva F, Specks U, Sethi S, Irazabal MV, Fervenza FC: Successful pregnancy and delivery of a healthy newborn despite transplacental transfer of antimyeloperoxidase antibodies from a mother with microscopic polyangiitis. Am J Kidney Dis 2009;54:542-545.

$\checkmark 5$ Talbot SF, Main DM, Levinson AI: Wegener's granulomatosis: first report of a case with onset during pregnancy. Arthritis Rheum 1984;27:109-112.

6 Piccoli GB, Mezza E, Bontempo S, Burdese M, Soragna G, Gai M, Consiglio V, Jeantet A, Segoloni GP, Piccoli G, Todros T: Vasculitis and kidney involvement in pregnancy: evidence-based medicine and ethics bear upon clinical choices. Nephrol Dial Transplant 2004;19:2909-2913.

7 Woywodt A, deGroot K, Bahte S, Schwarz A, Haller H, Haubitz M: Severe relapse of Wegener's granulomatosis during the early postpartum period. Ann Rheum Dis 2006; 65:137.
8 Harber MA, Tso A, Taheri S, Tuck SM, Burns A: Wegener's granulomatosis in pregnancy: the therapeutic dilemma. Nephrol Dial Transplant 1999;14:1789-1791.

-9 Dayoan ES, Dimen LL, Boylen CT: Successful treatment of Wegener's granulomatosis during pregnancy: a case report and review of the literature. Chest 1998;113:836-838.

10 Masterson R, Pellicano R, Bleasel K, McMahon LP: Wegener's granulomatosis in pregnancy: a novel approach to management. Am J Kidney Dis 2004;44:e68-e72.

11 Orbach H, Tishler M, Shoenfeld Y: Intravenous immunoglobulin and the kidney: a two-edged sword. Semin Arthritis Rheum 2004;34:593-601.
12 Stone JH, Merkel PA, Spiera R, Seo P, Langford CA, Hoffman GS, Kallenberg CGM, et al: Rituximab versus cyclophosphamide for ANCA-associated vasculitis. N Engl J Med 2010;363:221-232.

13 Jones RB, Cohen-Tervaert JW, Hauser T, Luqmani R, Morgan MD, Peh CA, Savage $\mathrm{CO}$, et al: Rituximab versus cyclophosphamide in ANCA-associated vasculitis. $\mathrm{N}$ Engl J Med 2010;363:211-220.

14 Vinet E, Pineau C, Gordon C, Clarke AE, Bernatsky S: Biologic therapy and pregnancy outcomes in women with rheumatic diseases. Arthritis Rheum 2009;61:587-592.

15 Østensen M, Lockshin M, Doria A, Valesini G, Meroni P, Gordon C, Brucato A, Tincani A: Update on safety during pregnancy of biological agents and some immunosuppressive anti-rheumatic drugs. Rheumatology $(\mathrm{Ox}-$ ford) $2008 ; 47:$ iii $28-$ iii 31 . 